The $M$ wave appared in order at level of nerve section from lower to upper. In that order, the strength-duration curves had also been returning from the right upper portion to normal curve level, however non recovered completely. They finally fixed on the relatively right upper portion in order at level of nerve sction from lower to upper group. By means of the supramaximal unishock, 2-3 phasic $\mathrm{M}$ wave was also smaller than normal.

Therefore it is concluded that the functional recovery was imperfect after the peripheral nerve section, and it could be expected poorer prognosis in the peripheral nerve section at higher level.

\title{
59. Clinical Studies of Phenol Block
}

\author{
Ichiro Fujita, Seiji KIshi, Emiko Soma and Takuhei KIDo \\ 1st Department of Surgery, Wakayama Medical College
}

In the 66th general congress of J.S.S., it was already reported that intractable pain might be relieved without norticeable side effects by injecting a dilute phenol solution into peripheral nerves. In order to examine possible changes in soft tissues histologically, the phenol solution was injected into the cutis and muscle, and around the vessel of rabbits. The results revealed the damages were little. We thus confident that the solution could be applied to clinical cases without troubles.

We tried the phenol block in 46 cases. That is to say, 26 cases with posttraumatic occipital pain and neuralgic pain in the 1 st branch region of trigeminus, 9 cases of the genuie trigeminal neuralgia, 7 cases of neoplasm and 4 miscellaneous cases. In more than $70 \%$ of all the cases subjected to the phenol block the procedure showed satisfactory effects.

\section{A New Method of Nerve Suture}

\author{
Takefumi Morotomi, Seiji Okazaki, Noboru Kawakami and Sanae Mizuta \\ Dept. of Orthopedics, Kyoto Prefectural Medical College
}

When the nerves are cut off, various methods of nerve sture must be done. Many of them, however, have weak points as well as merits. Generally speaking, the operation is difficult and takes too much time to be done. As we contrived a 